\title{
Contextual Research on Elderly Users' Needs for Developing Universal Design Mobile Phone
}

\author{
Hyunjeong Kim ${ }^{1}$, Jeongyun $\mathrm{Heo}^{2}$, Jungwha Shim ${ }^{1}$, Miyoung Kim ${ }^{1}$, Soojung Park ${ }^{1}$, \\ and Sanghyun Park ${ }^{2}$ \\ ${ }^{1}$ Graduate School of Digital Design, KyunSung University, 110-1 Daeyoun-dong, \\ Nam-gu, Busan, S. Korea \\ ${ }^{2}$ MC R\&D Center, LG Electronics, Kasan-Dong, Kumcheon-gu, Seoul, S. Korea \\ \{Hyunjeong Kim,kimhj\} astar.ks.ac.kr
}

\begin{abstract}
As the aged society and digital convergence have been progressed, 6 most of elderly users are having difficulties in using mobile phone with complex functions. It is necessary to develop mobile phone with easy and convenient usability for universal users including elderly based on concept of universal design. We conducted qualitative \& contextual research on elderly users' contextual experience and interaction difficulties in using mobile phone in everyday life. Based on elderly users' need figured out in this research, we tried to retrieve User Interface Design implication for universal design mobile phone, which can be used easily by anyone regardless of age and ability.
\end{abstract}

Keywords: contextual research, elderly user, universal design, mobile phone, UI design.

\section{Introduction}

As the age of digital convergence and ubiquitous computing has been arrived, the function of mobile phone has become more and more complex and various. Because mobile phone becomes more important and necessary in everyday life in Korea, the increased complexity causes severe inconvenience to people with physical or cognitive disabilities such as elderly users who are come up as a main consumer group by the progress of the aged society.

Therefore, it is necessary to develop mobile phone with easy and convenient usability for universal users including elderly. In order to develop universal mobile phone, research for elderly users' difficulties and needs in using mobile phone in everyday life should be preceded.

The objective of this research is to retrieve User Interface Design implication for universal design mobile phone, which can be used by anyone regardless of age and ability, through figuring out elderly users' difficulties and particularities in using mobile phone in everyday life. 


\section{Contextual Research Method}

\subsection{Characteristics of Contextual Research Method}

The first challenge of design is to understand the customers: their needs, their desires, and their approach to the work. Yet work becomes so habitual to the people who do it that they often have difficulty articulating exactly what they do and why they do it. Contextual inquiry uncovers who customers really are and how they work on a dayto-day basis. The cross-functional design team conducts one-on-one field interviews with customers in their workplace to discover what matters in the work. Team members observe people as they work and inquire into actions as they unfold to understand their motivations and strategy. The interviewer and customer, through discussion, develop a shared interpretation of the work.

Contextual research method is differentiated with usability test since it is a site visit to understanding how the client actually does. While usability test is a device-oriented evaluation process, contextual research method is a user-oriented discovery process. While usability test focuses the relationship between device or service and user, contextual research method focuses more on user environment including physical, psychological, cultural circumstances. Therefore, contextual researchers should have a wide perspective to look out over more various users' experience, not only interaction experience. Also, they should try to understand users' feelings and psychological problems more deeply and try to interpret cultural meaning of a device to users.

Team interpretation sessions bring the design team together to hear the whole story of each interview and capture the insights and learning relevant to their design problem. An interpretation session brings all team members' unique perspectives to the data, sharing design, marketing, and business implications. Through these discussions, the team captures issues, draws work models, and develops a shared view of the customer whose data is being interpreted and their needs.

\subsection{Contextual Research Process}

Survey for Recruiting Users. In order to recruit various level users, we implemented survey to 83 elderly people who uses mobile phone. The questions on survey were included to ask interest and level of usage on mobile phone. We selected 8 basic level users and 3 intermediary users over 60 based on survey result. Each level user can be categorized to the first groups who have intention to learn and use and the second group who give up to learn or who don't have intention to use.

The following table shows gender, age and model no. of the mobile phone, the participants were using.

Table 1. Profiles of elderly users

\begin{tabular}{lcc}
\hline Users & Don't have intention to learn \& use & Have intention to learn \& use \\
\hline \multirow{3}{*}{ Basic } & & Male, 61 yrs,SCH-X430 \\
Users & Female, 61yrs, LG SD330 & Male, 61 yrs, SPH-X4209 \\
& Male, 68 yrs, StarTAC 2004 & Male, 63 yrs, LG-SD9230 \\
& Male, 61 yrs, PS-K2500 & Male, 65 yrs, SPH-X4909 \\
Intermediary & Female, 72 yrs, SCH-X130 & Female, 67 yrs, SCH-E560 \\
level users & Male, 65 yrs, SPH-X4909 & Male, 69 yrs,SCH-X700 \\
\hline
\end{tabular}


The following figure shows contextual user research process applied in this study.

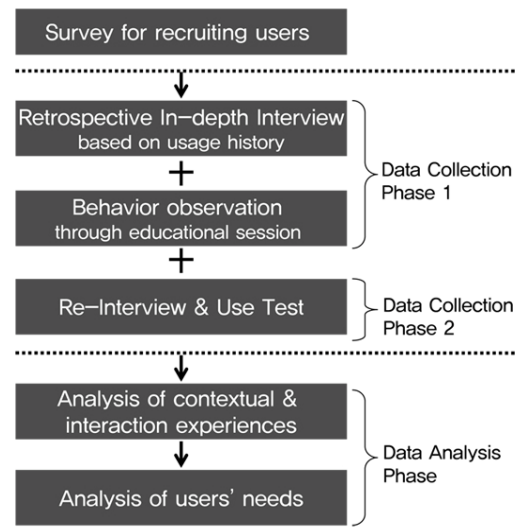

Fig. 1. Contextual research process applied in this study

Data Collection Method. First, we did retrospective in-depth interview to 11 elderly users regarding their perception of mobile phone, usage pattern and difficulties in using main functions in everyday life. Especially, we interviewed them with checking usage records (stored history on their phone) - in and out calls, sent and received text messages, phone number list on phone book, photo album, alarm and other settings. This method could help to inspect users' past experience with very concrete situation including usage context.

Second, we taught 8 basic level users with intention to learn major functionsinputting phone number on phone book, making a call by searching phone book, sending out text message, receiving and deleting text message, taking and saving a photo, checking and deleting photos. We intended to observe their perception, understandability, learnability, and error situation from their behavior during intimate, one-to one educational session.

Third, we re-interviewed and did usability test to 3 active users one month after the educational session, in order to examine how much and in what context they utilize newly learned functions in their everyday life, and what caused difficulties if they couldn't utilize them. The usability test could help to find error situation and analyze cause for the error.

All the interviews, educational sessions and tests were recorded under usability testing equipment to capture users' reaction and word and users' interaction with mobile phone.

Data Analysis Framework. Collected video data was reviewed and special episodes' video that shows elderly users' Contextual Experience (cultural, psychological factors) and Interaction Experience (difficulties in usage) were clipped. Each video clip was categorized into 7 usage situations - general perception/usage pattern, making/getting calls, using phone book, sending/getting text message, using camera, 

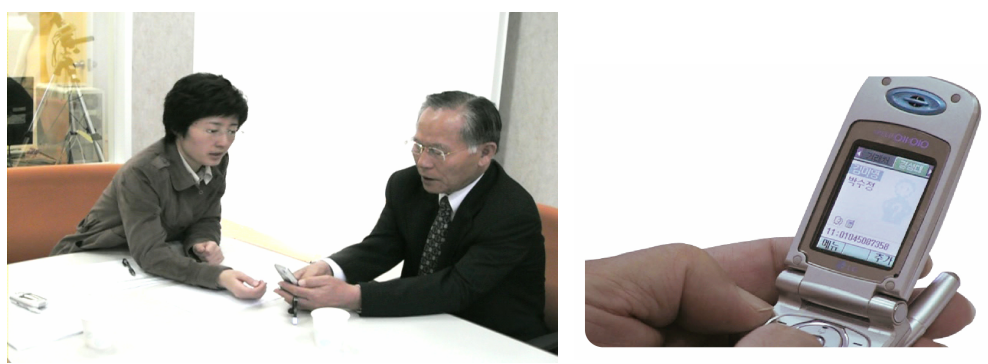

Fig. 2. Scenes from user interview \& interaction with mobile phone

using additional functions and et cetera. Almost 200 episode video clips were collected and each video clip was transcribed and added by observer's interpretation with notion of user factors and design factors. The following table 3 shows analysis form for episode video clips.

Table 2. Data Analysis Framework for episode video clips

\begin{tabular}{|c|l|l|}
\hline Situation & \multicolumn{2}{|l|}{ Category of 7 usage situation } \\
\hline $\begin{array}{c}\text { Contextual / } \\
\text { Interaction experience }\end{array}$ & \multicolumn{2}{|l|}{ Explanatory title for the episode } \\
\hline & & \\
& & \\
Observed Fact & Title of video clip \\
\hline Interpretation & Interpretation or note for the episode \\
\hline User factors & User related cause for the situation-physical, cognitive, and psychological \\
\hline Design factors & Design related cause for the situation \\
\hline
\end{tabular}

Tables to describe each episode were re-categorized to draw out categories of needs. Development directions or solution ideas for universal mobile phone were generated based on the interpretation of categorized, related situations (related episode video clips). The following chapter will describe the detail of the elderly users' needs in using mobile phone.

\section{Elderly Users' Needs in Using Mobile Phone and UI Design Implication}

Elderly users' needs were mainly categorized into 3 factors such as physical factors in product design, cognitive factors in UI design and cultural, psychological factors in usage context of mobile phone. 


\subsection{Physical Factors in Product Design}

\section{Need for vision}

Need for vision in screen. Elderly users were having vision difficulties in screen in the following cases; reading text in menu and text message especially when font is not gothic, and when color contrast between text and background is not obvious / identifying icons in upper part of the screen, whether text message is arrived or how much battery is left/ checking time in phone screen (they consider this feature very important) /identifying photo in album. They need bigger screen with big font size, gothic font type, obvious color contrast, obvious design or way to add text for status icons, big obvious clock setting, and a way to check photo with other big display equipment because of vision problem.

Need for vision in buttons. They were having vision difficulties in button in the following cases; identifying consonants and vowels of Korean and English on the button because they are layout so densely on small button/ finding side button's function. They need bigger button, separating design between number and text part on buttons, and more obvious clue for functions of the side buttons.

\section{Need for Hearing}

They missed calls because they couldn't hear ring tone and don't know how to change bell to vibration, so they selected the loudest ring tone, not the most favorable one. Many elderly users speak loud during call because they can't hear the counterpart. They need louder setting for ring tone with better speaker.

We found that a look of the mobile phone can influence to some elderly users' psychology regarding hearing. They associate better sound functionality to length that can cover from ear to mouth and to more or bigger holes on speaker. This misconception can be a reference to design appearance of mobile phone for elderly.

\section{Need for Manual Dexterity}

Need for manual dexterity in buttons. Elderly users become all thumbs, so we could find many times that they push wrong buttons (the very next button) or push buttons with finger nail because the button is too small and too close. They liked more obvious textual feedback and even auditory feedback by calling numbers. They prefer pushing less buttons. Especially, they couldn't control differentiating short push and long push on one button. They were also too slow when typing text message to complete a letter, because the cursor automatically moves to the next letter space. They need buttons with more obvious textual and auditory feedback when pushing buttons or navigating menus. Memorized number dialing should be provided with easy access. More than one function on one button should be restricted. Text making time should be set longer.

Need for manual dexterity in battery. They are having hard time when taking battery out or putting it in. The control part should be bigger and the manipulating way should be simple and clear. Graphic design on surface should give affordance to guide. 
Need for manual dexterity in camera. Elderly users often block lens with their fingers when they use camera first time. We found that many pictures were out of focus because their hands couldn't be still. For supporting manual dexterity in camera function, lens should be layout away from users' hand position, and it is better to have steady shot function and handler or finger supporting design.

Need for Convenient Carrying. Elderly users prefer small and lightweight phone because of power weakness. Specially, male users appeal for inconvenience to carry mobile phone when it is thick. Their hands are easy to slip things because grip power becomes weaker. They need thin, lightweight phone with bigger screen and button ironically. Male users need a way to carry phone more conveniently. They need better grip with non-slippery material and body design for grip support.

\subsection{Cognitive Factors in UI Design}

Need for Function Restriction vs. Usage Evolving. Elderly users need restrict functions to the extent that they often use. They feel that mobile phone has too many functions they don't use. They are interested in functions that are not related to their everyday life. They need that mobile phone should support well only its main functions such as phone call with phone book, text message, camera, clock and alarm and basic setting for screen theme and ring tone. However, some elderly users had more interests and questions regarding infrequently used menus after educational session.

Need for Alternative Method Restriction. During educational session, some elderly users couldn't understand the difference between making calls from phone book and from call history. They insist to use the first-time learned method even though there is more convenient way. Supplying alternative method made them confused. They don't want alternative methods when they learn first time. They need simplified and authentic method to learn simply and to remember easily.

Need for Feedback for Current Mode. Elderly users are having difficulties in identifying current mode, especially when the screen design looked similar such as phone book and call history or shooting mode and album mode. Some users were confused whether they were deleting text message or photo. They need more obvious clues or feedback where they are. The screen design should be distinctive when some pages could be looked alike. Deleting functions could have consistency.

Need for Easy Access (Shallow Depth). The major reason of failure for most tasks was the access failure. Elderly users couldn't find functions in navigating menu. They couldn't find where "input phone number" menu or "send message" menu is. They couldn't know where they change screen theme. They feel stressful when the depth becomes deep. On the other hand, they like direct call with memorized number button, even when they never understand phone book functions. They want frequent menus in shallow depth so that it can be accessed directly.

Need for Simplification of Process. The other major reason of failure was that process needed too many steps to finish. Some users can't send text message because she can't follow the steps to complete sending message out. Elderly users don't want 
to use voice mail because it needs annoying verification steps. Many elderly users understand phone book as to store a phone number per name. They couldn't finish inputting phone book because phone book page has so many rows to input such as address, e-mail, memo, photo and etc. They need simplified process with least pages, steps and options. Especially, voice mail should be more convenient so that it can replace the role of text message when elderly are too old to read text message in LCD screen.

Need for Clue What To Do Next. Elderly users can't recognize things unseen on the screen. They don't try scroll down to find menu 4 or 5 when the menu box shows up to 3 menus. They don't know way to check text message with left, right arrow keys. Some couldn't find shooting button when they are taking pictures. One of them didn't know what to do when alarm is ringing while the folder is close. On the contrary, another user didn't know what to do when phone is ringing while folder is open. They need clues what to do at the moment in the specific cases, or what to do next in the process.

Need for Task Oriented Label. Some label with English word, abbreviated word, Chinese word, or inconsistent word makes elderly users confused. They need Korean, task-oriented and consistent label with clear meaning.

Need for Text and Number Key than Icon. The most obvious distinction between elderly and young users was whether they navigate menu with number keys or with arrow keys. Most elderly users were using number keys. When the number key was gone or didn't work, elderly users were confused. They can't identify meaning of icons, so that number keys and text menu can be more efficient to deliver meaning. Especially, number key should be supplied anytime in any page and it shouldn't be gone or blocked.

\subsection{Cultural, Psychological Factors in Usage Context of Mobile Phone}

Need for Creating Usage Culture For Text Message And Camera Phone Among Elderly Users. Elderly users want to learn text message and how to take a picture with camera phone, but they say that there is no counterpart to send message and that they feel shy when using camera phone. It is social perception of them for mobile phone as exclusive possession of young people. If universal design phone for elderly users is developed, it should be promoted to create natural usage culture among elderly users.

Need for Normalization (Feeling Envious of High-End Function Phone). Most elderly users want to have high-end mobile phone even when they think they don't need high-end functions. Some users who have camera phone tend to show off their phone's ability. Some users who have outdated phones feel envious of high-end phone. Most of them feel negative for special function phone for elderly users exclusively. They said that they don't need complex functions, or special treatment as if they were old, handicapped people.

Need for Sparing Expenditure. Most elderly are very sensitive in saving expenditure because they experienced hard time in their young ages. Some of them 
were kind of obsessed by saving battery or charged electricity. They were also sensitive in saving calling rate. The quick charged and long lasting battery will be appeal to elderly users and saving cost plan should be supplied with hardware at the same time.

Need for Provision Against Emergencies. Elderly people are very cautious, so they need mobile phone to support emergencies such as when they are lost, when they have an accident, when weather becomes bad, and et cetera. They need someone else to call their home or family or hospital when they are unconscious. They need direct call function in emergency. They also want information service for abrupt weather change and where they are.

Need for Emotional Connection Or Self Verification. We found that many elderly users called missed calls back. They feel one call very precious because it is rare. They hesitate to call or mail to their sons and daughters, worrying they are not welcomed. Elderly users are likely to be lonely and want to be connected emotionally with family and friends, so that to verify their self-existence to others. Communication equipment should help to increase connectivity between people.

Preference to Old, Analog Things. Elderly users feel difficulties to adjust new equipment. Some of them insist to use same company's phone. Most of users bring memo pad, even when they knew how to use phone book inside of mobile phone. Most of them don't understand desktop metaphor in computer related product. The metaphor in mobile phone is targeted to young people. They prefer old, analog things in metaphor.

Need for Privacy. Some elderly users want to send secret text message only for recipient. It is universal need to be able to send secret message by sender.

\section{Development Strategy for Universal Design Mobile Phone}

Development strategy for universal design mobile phone can be divided into three categories based on target users and phone features. Silver phone has minimal HW features by eliminating features (barriers) elderly users do not use. Barrier Free Design concept is applied to this direction. Barrier free design pursues special solution to remove elderly or disabled people's difficulties. Silver phone targets only elderly users, so has minimal HW \& SW features that reflect elderly users' needs directly. Simple UI phone has minimal SW features and has simple UI that reflects elderly users' cognitive needs while it has high features such as Camera \& Color LCD. Universal Design concept is applied to Universal Design Phone. Universal design is the design of products and environments to be usable by all people, to the greatest extent possible, without the need for adaptation or specialized design The intent of universal design is to simplify life for everyone by making products, communications, and the built environment more usable by as many people as possible at little or no extra cost. Universal design benefits people of all ages and abilities. Therefore, considerations for elderly users are reflected on universal design phone to the extent that do not cause discomfort to young users. It targets universal users regardless of age and ability, while considering elderly users' needs. 


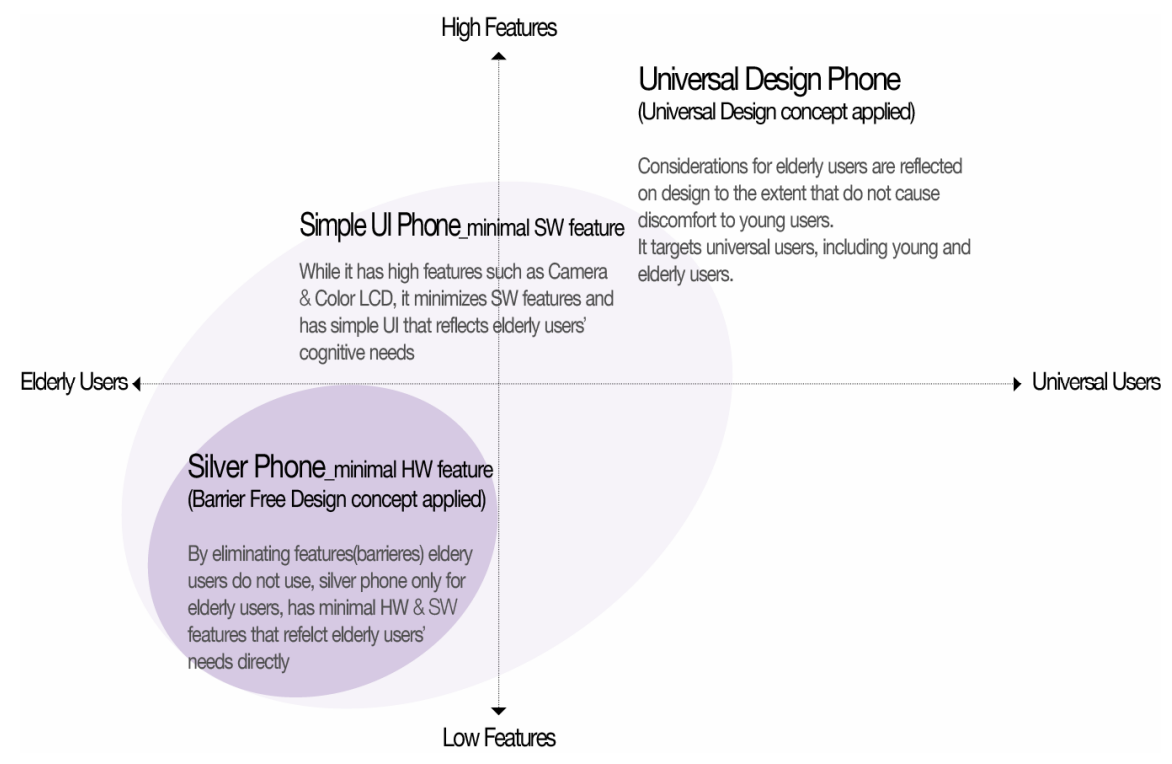

Fig. 3. Development strategy for universal design mobile phone

Elderly people's cognitive needs of eliminating functions to their frequently used functions can be taken care by supplying customization features to simplified menu in universal design mobile phone. Also, default setting should be simplified to fit to elderly users' behavior pattern, because young users can change settings easily. Appearance is very important factor in achieving universal design. The look should be targeted to young people or it should not have any generation orientation even though it has big buttons, big screen and large font.

It was suggested as the result of this research that development direction for universal design mobile phone in Korea should be Simple UI phone with simplified menu and UI based on elderly users' normalization needs.

\section{Conclusion}

The contextual and qualitative approach to elderly users makes to collect rich and substantial experience data in using mobile phone from their everyday lives. Especially, the episodes users describe their difficulties help to imagine the real situation vividly. Also, cultural, psychological understanding sought from contextual research makes to set the marketing strategy and to generate service ideas in addition to UI design implications.

This study shows a representative process of contextual research method that can be adapted to user experience research for mobile phone. The elderly users' need resulted from this research should be used to draw out UI design guidelines to develop universal design mobile phone. In this research, we select users from elderly people over 60. Since they are not accustomed to computer, they will be very 
different to computer-minded elderly users. In order to accomplish universal design mobile phone, the research to other groups of users including computer-minded elderly, children, teenagers, and the handicapped should be followed in the future.

Acknowledgments. We'd like to thank LG Electronics and Universal Design Research Center at KyungSung University to make this research possible.

\section{References}

1. Beyer, H., Holtzblat, K.: Contextual Design: A Customer-Centered Approach to Systems Designs. Morgan Kaufmann, New York (1997)

2. Kurniawan, S., Mahmud, M., Nugroho, Y.: A study of the use of mobile phones by older persons, CHI '06 (2006)

3. Kim, B.: A study on usability of mobile phone by elderly. In: A master's thesis, Hongik University, Seoul (2002)

4. Im, J., park, M.: Analysis of usability for universal design mobile phone with consideration of elderly users. Journal of the Ergonomics Society of Korea 24(1), 47-53 (2005)

5. Yamazaki, K., Matsuda, M., Yoshitake, R.: The concept and practice of User Centered Design, Maruzen Co., Ltd. Tokyo (2004)

6. Universal Design KENKYUKAI, Shin-Universal Design, Japan Industrial Publishing Co., Ltd. Tokyo (2005) 